\title{
Concomitant treatment with oral L-arginine improves the efficacy of surgical angiogenesis in patients with severe diffuse coronary artery disease: The Endothelial Modulation in Angiogenic Therapy randomized controlled trial
}

Marc Ruel, MD, MPH, ${ }^{\mathrm{a}, \mathrm{b}}$ Robert S. Beanlands, MD, ${ }^{\mathrm{c}}$ Mireille Lortie, PhD, ${ }^{\mathrm{c}}$ Vincent Chan, MD, ${ }^{\mathrm{a}}$ Nancy Camack, RN, ${ }^{\mathrm{d}}$ Robert A. deKemp, PhD, ${ }^{c}$ Erik J. Suuronen, PhD, ${ }^{a}$ Fraser D. Rubens, MD, ${ }^{a}$ Jean N. DaSilva, PhD, ${ }^{\mathrm{c}}$ Frank W. Sellke, MD, Duncan J. Stewart, MD, ${ }^{d}$ and Thierry G. Mesana, MD, PhD ${ }^{a}$

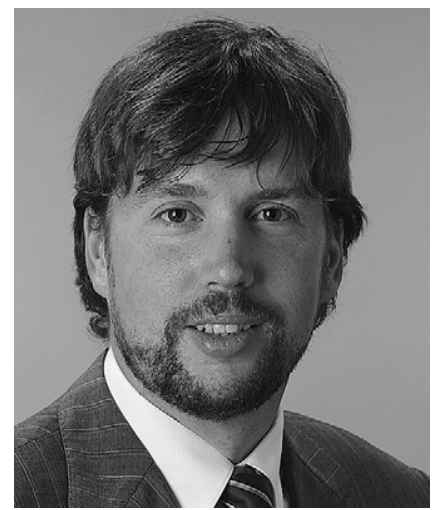

Dr Marc Ruel

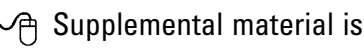
available online.

From the Division of Cardiac Surgery, ${ }^{\mathrm{a}}$ Department of Epidemiology, and Division of Cardiology, ${ }^{\mathrm{c}}$ University of Ottawa, Ottawa, Ontario, Canada; Division of Cardiology, ${ }^{\mathrm{d}}$ University of Toronto, Toronto, Ontario, Canada; and Department of Surgery, ${ }^{\mathrm{e}}$ Harvard Medical School, Boston, Massachusetts.

Funded by grant NA5163 from the Heart and Stroke Foundation of Canada (M. Ruel). Duncan Stewart reports equity ownership of Northern Therapies, the manufacturer of the growth factor used in this study.

Read at the Eighty-seventh Annual Meeting of The American Association for Thoracic Surgery, Washington, DC, May 5-9, 2007.

Received for publication May 4, 2007; revisions received Sept 16, 2007; accepted for publication Sept 25, 2007.

Address for reprints: Marc Ruel, MD, MPH, University of Ottawa Heart Institute, 40 Ruskin Street, Suite 3403, Ottawa, Ontario, Canada K1Y4W7 (E-mail: mruel@ottawaheart.ca).

J Thorac Cardiovasc Surg 2008; 135:762-70 $0022-5223 / \$ 34.00$

Copyright (๑) 2008 by The American Association for Thoracic Surgery

doi:10.1016/j.jtcvs.2007.09.073
Objective: Endothelial dysfunction and decreased nitric oxide bioavailability may explain why therapeutic angiogenesis and cell therapy have mostly failed in humans. Building from previous large animal work, the Phase I Endothelial Modulation in Angiogenic Therapy trial tested the hypothesis that L-arginine, a nitric oxide donor, may be safe and effective in potentiating surgical angiogenesis in humans.

Methods: Patients with surgical triple-vessel coronary disease and a severely diffusely diseased left anterior descending artery were randomized in $2 \times 2$ factorial fashion to receive ten $200-\mu \mathrm{g}$ injections of vascular endothelial growth factor-165 plasmid DNA or placebo in the anterior myocardium along the proximal and mid-left anterior descending arteries, plus oral L-arginine supplementation at a dose of $6 \mathrm{~g}$ per day or placebo for 3 months. The distal left anterior descending artery and other coronary arteries were grafted. End points included 3-month changes in myocardial perfusion and contractility of the anterior myocardium, using ${ }^{13} \mathrm{~N}$-ammonia positron emission tomography and echocardiography. Baseline scans were obtained 3 to 7 days postoperatively to delineate treatment effects from the effects of coronary artery bypass grafting.

Results: Patient $(\mathrm{N}=19)$ characteristics were equivalent between groups. There was no perioperative or late mortality. Patients who received the combination of vascular endothelial growth factor and L-arginine had improved anterior wall perfusion on positron emission tomography $(P=.02)$, a trend toward smaller perfusion defects $(P=.10)$, and better anterior wall contractility $(P=.02$, Kruskal-Wallis $)$ at 3 months versus baseline. This was corroborated by a trend toward better disease perception at 3 months versus baseline on the Seattle Angina Questionnaire (score improvement of $47 \pm 35$, combination treatment group; $P=.1$, Kruskal-Wallis).

Conclusion: To our knowledge, this is the first study to examine concomitant substrate modification in patients undergoing new biosurgical therapies by using vascular endothelial growth factor angiogenesis. The results suggest safety and efficacy. Concomitant endothelial modulation with L-arginine not only has the potential to make angiogenesis effective but also may have implications for cell therapy trials.

$\mathrm{P}$ atients with coronary artery disease (CAD) have markedly abnormal coronary endothelial function and decreased nitric oxide (NO) bioavailability, particularly when the CAD is severe and diffuse. ${ }^{1-4} \mathrm{We}$ and others have shown that angiogenesis depends on local NO levels, ${ }^{5-7}$ which may explain the discrepancy between successful animal studies and disappointing clinical trials of therapeutic 


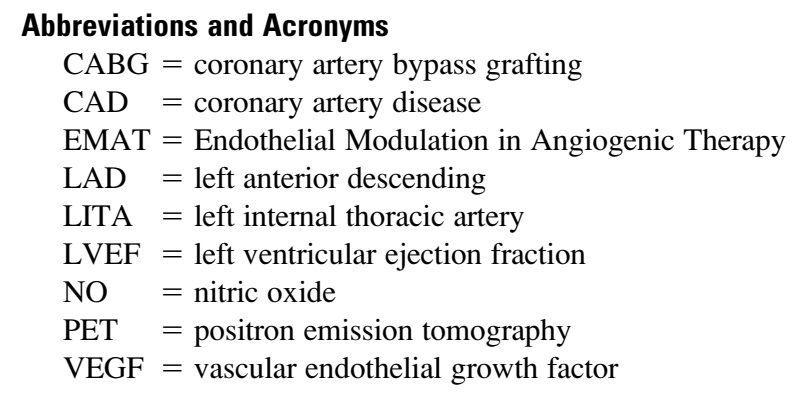

angiogenesis. ${ }^{8}$ Furthermore, because paracrine angiogenesis is a proposed mechanism for the effects of myocardial cell therapy, ${ }^{9,10}$ it is possible that endothelial dysfunction and decreased NO bioavailability may explain why cell therapy trials have so far also led to disappointing results.

In animals, dietary supplementation of L-arginine, the substrate for NO production, has been shown to reverse the inhibition of angiogenesis that results from endothelial dysfunction. ${ }^{11-13}$ Because L-arginine has been shown to improve coronary endothelial dysfunction in humans, ${ }^{14-17}$ we tested the hypothesis that its use in patients undergoing angiogenic therapy may make angiogenesis clinically more effective.

The Endothelial Modulation in Angiogenic Therapy (EMAT) trial is a phase I $2 \times 2$ factorial, double-blind, double placebo-controlled trial designed to evaluate the safety and efficacy of intramyocardial vascular endothelial growth factor (VEGF)-165 angiogenesis combined with oral L-arginine supplementation in patients with severe diffuse CAD undergoing coronary artery bypass grafting (CABG). The primary end point of the trial was target territory myocardial blood flow reserve quantified by positron emission tomography (PET). Secondary end points were target territory contractility on echocardiography, ischemic zone size on PET, and clinical outcomes.

\section{Materials and Methods Patient Enrollment}

The trial was approved by Health Canada, and research ethics board approval was granted by the University of Ottawa. The trial was registered at ClinicalTrials.gov as NCT00134433. Enrollment took place between August 12, 2004, and May 8, 2006. Patients were at least 18 years of age, had symptomatic multivessel CAD, and had been identified by at least 1 non-investigator cardiologist and surgeon as having severe, diffuse CAD in the left anterior descending (LAD) artery ( \pm other coronary vessels) that could compromise feasibility of surgical revascularization. Exclusion criteria included pregnancy, lactation, or child-bearing potential; amenability for percutaneous coronary intervention; akinesis of the anterior wall; left ventricular ejection fraction (LVEF) less than $30 \%$; recent $(<1$ month) ST-elevation myocardial infarction; chronic renal failure (serum creatinine $>130 \mu \mathrm{mol} / \mathrm{L}$ ); hepatic insufficiency (ChildPugh Class $\mathrm{C}$ or more); clinically significant valvular heart disease; personal history of neoplasia; abnormal bowel cancer screening questionnaire result; abnormal serum prostate specific antigen (if male) or abnormal mammography (if female); family history of cancer in more than 1 first-degree relative; diabetic retinopathy; latent herpes infection; schizophrenia; and claustrophobia.

Twenty-six eligible patients were referred among 282 patients who underwent isolated primary CABG referred to the principal investigator (M.R.) during the enrollment period. Seven of these 26 eligible patients refused enrollment. One patient was operated by another surgeon (F.R.) with the principal investigator in attendance during the study procedures. Overall, 19 patients provided written informed consent, were enrolled and randomized, and underwent study procedures and follow-up.

\section{Randomization}

At operation, diffuse distal CAD of the target territory was confirmed intraoperatively by a non-investigator surgeon before final confirmation of eligibility. If eligible, each patient was double-randomized to 1 of 4 groups: 1) placebo intramyocardial injections intraoperatively + placebo oral supplementation postoperatively; 2) VEGF intramyocardial injections intraoperatively + placebo oral supplementation postoperatively; 3) placebo intramyocardial injections intraoperatively + L-arginine oral supplementation postoperatively; or 4) VEGF intramyocardial injections intraoperatively + L-arginine oral supplementation postoperatively. The randomization was doublestratified by gender and according to the presence of LV dysfunction (LVEF $<40 \%$ ). Patients, their families, non-investigator clinicians, and all members of the research team with the exception of the research pharmacist were blinded to the treatment group assignment.

\section{Surgical Technique and Postoperative Procedures}

All operations were performed through a median sternotomy with the use of cardiopulmonary bypass, aortic crossclamping, and antegrade cold blood cardioplegia delivered at 20-minute intervals during cardiac anoxia. Patients had the left internal thoracic artery (LITA) grafted onto the LAD at a target site selected as being the most proximal section of the LAD that was free of stenosis more than $50 \%$ distally. This site was determined by 2 surgeons (different from the surgeon involved in patient screening/identification) from the combination of angiography and epicardial examination of the vessel, and consisted of the distal LAD in all patients. At least 1 additional arterial conduit (consisting of the right internal thoracic artery or left radial artery) was used to graft the target vessel from the circumflex or right coronary distribution that subjectively subtended the largest myocardial territory on coronary angiography. Greater saphenous vein segments were used to complete the revascularization, including the diagonal system if well developed and suitable for bypass.

Before release of the aortic crossclamp after completion of the last distal anastomosis, 10 injections containing either good manufacturing practice-grade, Health Canada-approved VEGF-165 plasmid DNA (pVAX-1 VEGF165; manufactured at St Michael's Hospital) at a dose of $200 \mu \mathrm{g}$ per injection for a total of $2 \mathrm{mg}$ (total injected volume $1.8 \mathrm{~mL}$ ), or physiologic saline (using an equal volume and number of injections) were performed $1 \mathrm{~cm}$ apart, in the underlying anterior septum and myocardium along the proximal and midLAD arteries ( \pm the initial distal LAD territory if proximal to the anastomosis) cephalad to the LITA-distal LAD anastomotic site. No injections were performed distal to the LITA-distal LAD 
anastomosis, because the anastomosis was performed at a site where the downstream LAD was free of angiographically significant $(>50 \%)$ stenosis. The remainder of the operation was performed as per routine. The study's first patient, who had been randomized to placebo injections and had diffuse disease of the posterior descending artery in addition to diffuse LAD disease, had a LITA-distal LAD anastomosis and received inferior wall (placebo) injections along the diseased posterior descending artery. After this initial patient, the protocol was amended to restrict it to injections along the diffusely diseased proximal and mid-LAD arteries only.

Once in the cardiac surgical recovery unit, all patients were given the contents of 1 orange-flavored sachet containing either $6 \mathrm{~g}$ of Larginine (Unither Pharmaceuticals, Silver Spring, Md) or placebo reconstituted in $125 \mathrm{~mL}$ of water, administered via nasogastric tube 4 hours after operation. On subsequent days throughout the 3 -month study period, sachets were reconstituted in $250 \mathrm{~mL}$ of water and taken daily by mouth.

Three to 5 days after CABG ( 7 days in 1 patient), patients underwent nuclear rest/stress ${ }^{13} \mathrm{~N}$-ammonia PET to determine baseline myocardial perfusion and ischemic zone size, as well as transthoracic echocardiography to assess baseline anterior and global LV function. Baseline imaging was performed during the early postoperative period rather than preoperatively to better separate the treatment effects from the effects of CABG. ${ }^{18}$ Follow-up PET and transthoracic echocardiography were repeated 3 months later at the completion of the study. PET and echocardiography data were interpreted by an experienced nuclear cardiologist and echocardiographer, respectively, who were both blinded to treatment and other clinical and imaging data. Other aspects of postoperative care were carried out as per routine.

\section{Myocardial Perfusion}

The primary end point of the study was differences in anterior myocardial territory blood flow reserve quantified by PET. To this end, patients underwent rest and dipyridamole stress ${ }^{13} \mathrm{~N}$-ammonia dynamic PET imaging at baseline and at 3 months' follow-up. Scans were acquired on the ECAT ART (Siemens/CTI, Knoxville, Tenn) PET scanner $(12 \times 10$ seconds, $2 \times 30$ seconds, $1 \times 60$ seconds, $1 \times 120$ seconds, $1 \times 240$ seconds, $2 \times 300$ seconds, total $=20$ minutes). Dynamic images were reconstructed using filtered back-projection with a 12-mm Hann window and automatically reoriented into short-axis sections. ${ }^{19}$ Maximum activity profiles were obtained from the uptake image, with a combination of planar and conical sampling, and used to define the 3-dimensional shape of the LV myocardium. ${ }^{20}$ The resulting 496 mid-myocardial coordinates were used to resample the short-axis images into dynamic polar maps, producing regional time-activity curves for the myocardium. The arterial blood time-activity curve was obtained from the average of 12 short-axis image regions $\left(1.4 \mathrm{~cm}^{2}\right.$ each), automatically placed in the center of the cavity and base regions of the $\mathrm{LV}$, and in the center of the left atrium. A first-pass model was used to calculate the net retention rate, with software corrections for wall thickness and motion.

Flow reserve was defined as the ratio of stress retention over rest retention. Polar maps were used to calculate segmental LV values, which were analyzed by using automated computer software to provide independent, unbiased data. The anterior wall (4 subsegments) was the target myocardial territory examined for evidence of new angiogenesis through increased flow reserve. The mid-inferior wall (2 subsegments), remote from intramyocardial injections, was used as a reference/control myocardial territory. The patient who received inferior wall injections was excluded from all regional myocardial perfusion analyses.

\section{Left Ventricular Contractility}

Dedicated study transthoracic echocardiograms were performed at postoperative baseline and 3 months' follow-up with the patient placed in a left lateral decubitus position. Parasternal long- and short-axis, and apical 2- and 4-chamber views were digitally acquired and recorded for subsequent analysis. The left ventricle was divided into 16 segments, ${ }^{21}$ and a regional wall motion score was used (normal $=1$, hypokinetic $=2$, akinetic $=3$, dyskinetic $=4$ ). The anterior wall scores at postoperative baseline were subtracted from the corresponding scores at 3 months' follow-up to isolate the interval changes in anterior wall contractility. The patient who received inferior wall injections was included in the contractility analyses because he presented severe diffuse LAD disease, had a LITA-distal LAD anastomosis, and received placebo.

\section{Clinical Outcomes}

The secondary end points of the trial included major adverse cardiac events, freedom from angina, angina class, and Seattle Angina Questionnaire scores at 3 months versus preoperatively. All patients were contacted by the investigators at 1,2, 3, and 6 months; 1 year; and yearly thereafter for a period of 15 years as per Health Canada requirements. Clinical follow-up for more than 9 months is available for all patients, and the mean follow-up was $16.7 \pm 6.8$ months.

\section{Power Calculations and Statistical Analyses}

This phase I trial was designed to detect a myocardial blood flow difference of $0.25 \mathrm{~mL} / \mathrm{min} / \mathrm{g}$ between study groups on PET, at an $\alpha$ error level of 0.0125 and with a power of 0.80 , with respect to the combination of VEGF and L-arginine versus control groups (ie, no active agent or either single agent). The $0.25 \mathrm{~mL} / \mathrm{min} / \mathrm{g}$ difference was chosen as within the reported myocardial flow and flow reserve responses to clinically beneficial treatments, which have been reported as ranging from $12 \%$ to $54 \%$, assuming a normal resting flow of $1.0 \mathrm{~mL} / \mathrm{min} / \mathrm{g} .{ }^{22}$ On this basis, the planned sample size was 9 subjects per group $\times 4$ groups $=36$ subjects.

Discrete data are presented as numbers and percentages, and continuous data are presented as mean \pm standard deviation or median. Because of their normal distribution, determined by the ShapiroWilk W test, PET flow reserve and defect size data were compared by using analysis of variance (at baseline, follow-up, and their difference). All other data, including clinical and echocardiography data, were compared by using a Kruskal-Wallis test. All reported probability values were 2-tailed. Intercooled Stata version 9.2 (Stata, College Station, Tex) was used for the statistical analyses.

\section{Early Study Termination}

On May 16, 2006, Health Canada released a public advisory stating that "a recent study published in the Journal of the American Medical Association in January of 2006 suggests that L-arginine may not help improve heart and circulatory function following a first heart attack and may be associated with an increased risk of death when used after a heart attack. ${ }^{23}$ All L-arginine products must carry a warning on their label that reflects the recent scientific information. Health Canada has issued a stop-sale and recall on L-arginine- 
containing products that do not have the appropriate labeling. (...) For patients with heart disease who have not had a previous heart attack, taking L-arginine is unlikely to present a risk and may provide benefits since L-arginine may help the body repair damage to blood vessels in the heart."

As of May 16, 2006, 19 patients had been enrolled in the EMAT trial. All patients had successfully undergone operation, and the last 3 patients were still taking postoperative daily oral L-arginine or placebo supplementation. We confirmed with Health Canada that the advisory did not apply to patients in the EMAT trial, and a letter was sent to the 3 patients who were still taking oral supplementation, explaining the advisory and stressing that they were free to stop taking the L-arginine or placebo. All 3 patients chose to continue taking the daily supplementation until the completion of the study. The trial's Data Safety and Monitoring Board reviewed all available data and reported no related major adverse event. We decided to stop enrollment for ethical reasons, with the study's last patient consequently having undergone operation on May 8, 2006, before the advisory.

\section{Results}

\section{Patient Characteristics and Perioperative Results}

The results of the randomization schedule are available as supplemental online material (Table E1). Baseline patient characteristics were similar between groups (Table 1). No patient had left main coronary stenosis greater than $60 \%$. The extent of CABG surgery was the same in all treatment groups, and coronary endarterectomy was avoided in all patients. Intravenous tranexamic acid was administered to all patients during the operation. There were no significant differences between groups with regard to the number of grafts (average $3.1 \pm 0.2$ ), number of arterial conduits (average
$2.4 \pm 0.2$ ), duration of cardiopulmonary bypass (average 80 \pm 21 minutes), or crossclamp time (average $53 \pm 17$ minutes).

Patients received titrated intravenous nitroglycerin $(0-2$ $\mu \mathrm{g} / \mathrm{kg} / \mathrm{min})$ and/or sodium nitroprusside $(0-1 \mu \mathrm{g} / \mathrm{kg} / \mathrm{min})$ during the first 24 postoperative hours if they were hypertensive or if a radial artery graft had been performed. Dipyridamole was not used at any time other than during PET imaging.

There was no early or late postoperative mortality. The times to extubation (mean $11.7 \pm 7.0$ hours; median 8 hours), discharge from the intensive care unit $(1.6 \pm 1.1$ days; median 1 day), and discharge from hospital (10.4 \pm 8.7 days; median 8 days) were not different between groups. There was no reopening for bleeding, perioperative myocardial infarction (by myocardial enzyme, electrocardiography, or echocardiographic criteria), reintubation, or readmission to hospital.

Postoperatively, all patients were maintained on aspirin, an angiotensin-converting enzyme inhibitor or angiotensin receptor blocker, a cholesterol-lowering agent, and a betablocker. Three patients resumed their preoperative oral nitrate medication (one in each of the VEGF + placebo oral supplementation, placebo injections + L-arginine, and VEGF + L-arginine groups). All patients who received a radial artery graft were prescribed oral amlodipine $2.5 \mathrm{mg}$ daily for a period of 6 months.

\section{Clinical Follow-up}

At last follow-up, all patients were alive, showed Canadian Cardiovascular Society angina class 1 or 2 , and had no myocardial infarction. There was no difference between groups with respect to these outcomes. The second patient

TABLE 1. Baseline patient characteristics

\begin{tabular}{|c|c|c|c|c|c|}
\hline & $\begin{array}{l}\text { All patients } \\
(\mathrm{n}=19)\end{array}$ & $\begin{array}{l}\text { Placebo/placebo } \\
\quad(n=1)\end{array}$ & $\begin{array}{c}\text { VEGF/placebo } \\
(n=7)\end{array}$ & $\begin{array}{l}\text { Placebo/L-arginine } \\
\qquad(n=6)\end{array}$ & $\begin{array}{l}\text { VEGF/L-arginine } \\
\quad(n=5)\end{array}$ \\
\hline Age at surgery, y & $59.1 \pm 8.6$ & 66 & $58.3 \pm 9.7$ & $60.0 \pm 8.7$ & $57.8 \pm 8.9$ \\
\hline Men/women, $\mathrm{n}$ & $18 / 1$ & $1 / 0$ & $6 / 1$ & $6 / 0$ & $5 / 0$ \\
\hline Body surface area, $\mathrm{m}^{2}$ & $2.1 \pm 0.2$ & 2.3 & $2.1 \pm 0.2$ & $2.1 \pm 0.1$ & $2.2 \pm 0.2$ \\
\hline Hypertension, $n$ & $14(74 \%)$ & 0 & 6 & 4 & 4 \\
\hline Tobacco use, $\mathrm{n}$ & $3(16 \%)$ & 0 & 1 & 1 & 1 \\
\hline Hypercholesterolemia, $n$ & $16(84 \%)$ & 0 & 5 & 6 & 5 \\
\hline Diabetes, $\mathrm{n}$ & $10(53 \%)$ & 1 & 4 & 3 & 2 \\
\hline \multicolumn{6}{|l|}{ CCS angina class } \\
\hline 1 & - & - & - & - & - \\
\hline 2 & $3(16 \%)$ & - & 1 & 2 & - \\
\hline 3 & $9(47 \%)$ & - & 4 & 2 & 3 \\
\hline 4 & $7(37 \%)$ & 1 & 2 & 2 & 2 \\
\hline \multicolumn{6}{|l|}{ Left ventricular grade ${ }^{*}$} \\
\hline 1 & $12(63 \%)$ & - & 4 & 4 & 4 \\
\hline 2 & $4(21 \%)$ & - & 2 & 2 & - \\
\hline 3 & $3(16 \%)$ & 1 & 1 & - & 1 \\
\hline 4 & - & - & - & - & - \\
\hline
\end{tabular}

VEGF, Vascular endothelial growth factor; CCS, Canadian Cardiovascular Society. *Grade 1, LVEF $\geq 50 \%$; grade 2, ejection fraction $40 \%$ to $49 \%$; grade 3, ejection fraction $30 \%$ to $39 \%$; grade 4 , ejection fraction $<30 \%$. 
enrolled in the trial underwent coronary and graft angiography 3 months postoperatively for asymptomatic perfusion abnormalities on PET. At angiography, grafts were found to be widely patent, and the proximal and mid-LAD arteries, which had been noted to be diffusely obliterated at operation, appeared angiographically reconstituted. The patient, who after completion of the study was identified as having received VEGF + L-arginine, has been free of angina since the operation and works full time. For all other patients in the EMAT trial, we based the decision to perform repeat coronary angiography on the presence of clinical symptoms, and no other patient underwent repeat angiography at the last follow-up.

There was no difference in angina severity on the Seattle Angina Questionnaire between groups. An improvement of $47 \pm 35$ in disease perception scores at 3 months versus preoperatively was observed in patients who received the combination of VEGF + L-arginine, versus $17 \pm 29$ during the corresponding period in patients from other groups $(P=.1$, Kruskal-Wallis).

\section{Myocardial Perfusion}

Figure 1 (top) displays the myocardial flow reserve in a control myocardial territory (the mid-inferior wall) and in the target myocardial territory (the anterior wall) on ${ }^{13} \mathrm{~N}$-ammonia PET at baseline and follow-up, according to treatment group. The patient who had placebo injections to the inferior myocardium was excluded from these analyses. There was no difference in flow reserve in the mid-inferior myocardial territory at any time point between groups; however, there was increased flow reserve at 3 months' follow-up in the anterior wall in the group who received VEGF + L-arginine (middle). This was observed both as a higher flow reserve at follow-up and as a trend toward a significantly greater difference in flow reserve at followup from baseline. The increase in anterior wall flow reserve from baseline to follow-up was also seen in the VEGF + L-arginine combination group when compared with patients who received either or both placebos $(P \leq .02$; bottom). Figure 2 depicts anterior wall stress perfusion abnormalities at follow-up in a patient who had received VEGF + placebo versus a patient who had received VEGF + L-arginine.

Figure 3 displays the changes between baseline and follow-up in the size of the rest and stress anterior myocardial wall defects as measured by PET. In the VEGF + L-arginine combination group, both rest and stress anterior wall defects decreased in size, whereas the reverse was observed in the groups who received either or both placebos. This was associated with a trend toward statistical significance $(P=.1)$.

\section{Left Ventricular Contractility}

LVEF increased by an average of $6.6 \% \pm 5.3 \%$ from preoperatively to 3 months' follow-up and by $1.6 \% \pm 7.3 \%$ from postoperative baseline to 3 months' follow-up. There were no significant differences in LVEF or LVEF change between

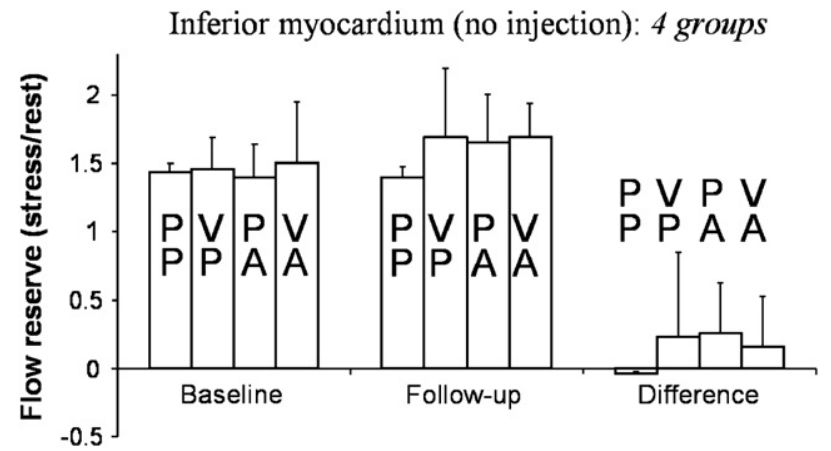

Anterior myocardium (injections): 4 groups

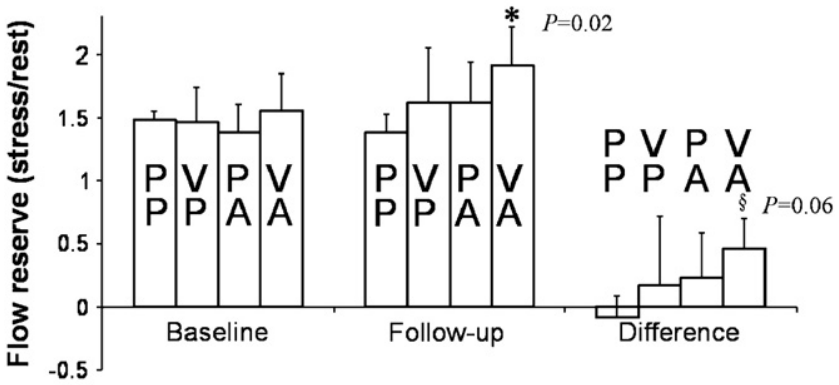

Anterior myocardium (injections): all placebo groups $(\mathrm{P})$ vs. combination group $(\mathrm{C})$

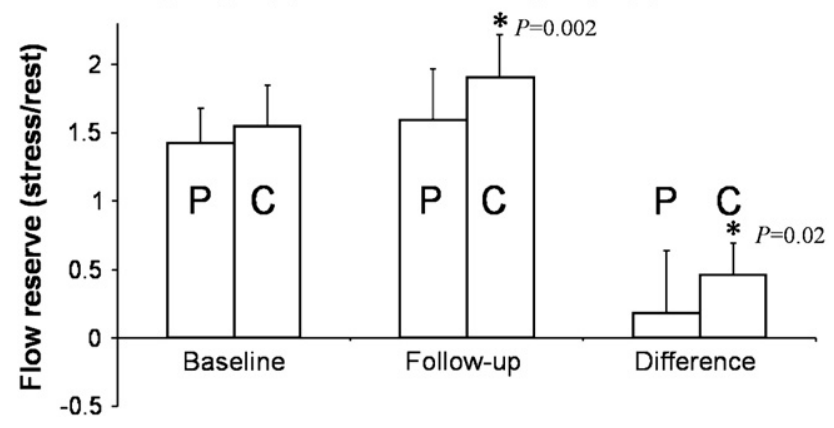

Figure 1. Myocardial flow reserve in a remote, noninjected myocardial territory (mid-inferior wall) and in the injected myocardial territory (the anterior wall) on ${ }^{13} \mathrm{~N}$-ammonia PET at baseline and 3-month follow-up. Treatment groups are denoted as $\mathbf{P}$ (either or both placebos), V (VEGF), A (L-arginine), and C (combination of VEGF + L-arginine). There was no difference in flow reserve in the noninjected (mid-inferior) myocardial territory at any time point between groups (top); however, there was increased flow reserve at 3 months' follow-up in the injected (anterior) myocardium of patients who received VEGF + L-arginine (middle). This was observed on flow reserve at follow-up and by a trend toward a significantly greater difference from baseline flow reserve. The increase in anterior myocardial flow reserve in the VEGF + L-arginine combination group from baseline to follow-up was also seen when this group was compared with all other groups (bottom). 

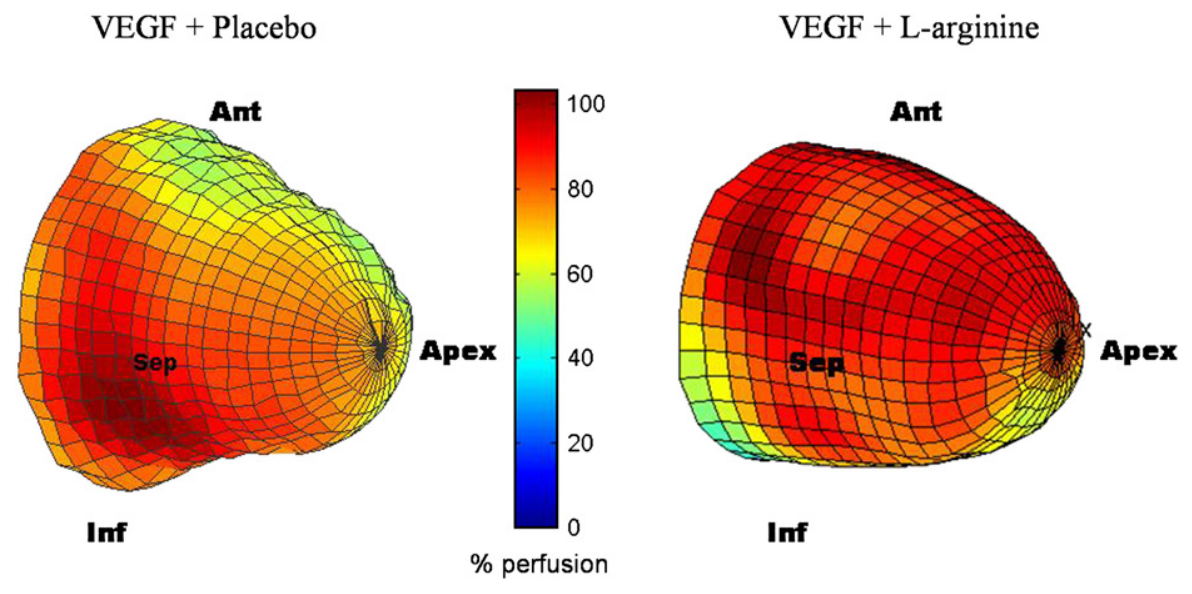

Figure 2. Three-dimensional left ventricular representation of stress perfusion at 3 months' follow-up in a patient who received VEGF along the proximal and mid-LAD arteries + daily placebo oral supplementation for 3 months (left) versus a patient who received VEGF + daily L-arginine oral supplementation for 3 months (right). VEGF, Vascular endothelial growth factor; Ant, anterior wall; Inf, inferior wall; Sep, septum.

groups. In the anterior wall, the contractility at 3 months' follow-up versus postoperative baseline showed mild improvement in 1 patient and no change in 4 patients who received the VEGF + L-arginine combination, versus no change in 7 patients and a mild deterioration in 7 patients who received either or both placebos ( $P=.02$, Kruskal-Wallis). There was no significant difference between groups in the contractility of other LV segments.

\section{Discussion}

The results of the EMAT trial suggest that the combination of intramyocardial VEGF and L-arginine oral supplementation for 3 months is safe and may potentiate angiogenesis. Although the trial was stopped early, there is a suggestion of efficacy for the primary end point of flow reserve in the anterior myocardium, which was significantly higher in patients who received VEGF and L-arginine versus those who received either or both placebos. This was independent from the effects of CABG and was corroborated with a trend toward better Seattle Angina Questionnaire disease perception scores at 3 months versus baseline.

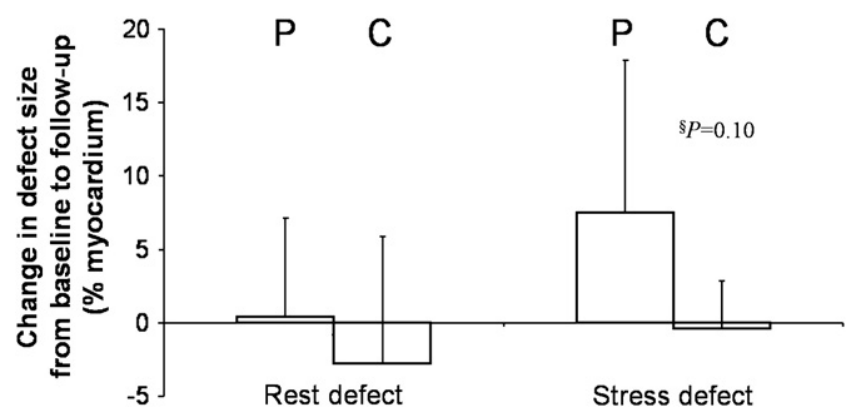

Figure 3. Changes in rest and stress defect size between baseline and 3 months' follow-up, as measured by ${ }^{13} \mathrm{~N}$-ammonia PET. The defects decreased in size in the VEGF + L-arginine combination group (C), whereas the reverse was observed in groups who received either or both placebos $(P)$.
Another finding of the EMAT trial is that VEGF and L-arginine were not associated with major adverse events. Although this is only suggestive of safety because the number of patients was small, this is nevertheless important considering the recent concern surrounding L-arginine use in patients immediately after myocardial infarction; ${ }^{23}$ in light of the present findings, it may be advisable to not hastily rule out all future research using L-arginine in humans with CAD because of that single trial. In fact, L-arginine has been shown to be clinically safe, to have beneficial effects as a NO donor, and to play a role in patients not immediately post-myocardial infarction when administered in a carefully controlled setting.

In this trial, we selected VEGF as an angiogenic agent because it is well studied, safe, and clinically available, and has a dependency on local NO availability that may exceed that of other angiogenic agents, such as fibroblast growth factor-2. ${ }^{24}$ VEGF therefore seemed well suited for our study hypotheses. It is noteworthy that the LAD was bypassed in all patients enrolled in this trial, distally as mandated by the diffuse distal involvement, to "protect" them against the possibility of ineffective angiogenesis. This shifted the focus of the trial on regional myocardial perfusion differences within the intervened myocardial territory rather than on differences in clinical outcomes; therefore, the trial was powered on a physiologic rather than a clinical end point. Consequently, clinical and perfusion differences were expectedly small, because all patients received multi-arterial, complete revascularization. To convincingly establish the clinical role of concomitant endothelial modulation therapy in combination with new biosurgical therapies, subsequent trials that evaluate their interaction will need to be adequately powered to show a difference in clinical outcomes.

We do not believe that VEGF + L-arginine combination therapy should be offered to patients with diffuse distal CAD solely on the basis of the results of this trial. However, the present study provides data that are relevant and 
hypothesis-generating to help explain why angiogenesis and gene therapy have so far largely failed in patients, and why clinical cell therapy may continue to lead to disappointing results until host factors such as coronary endothelial dysfunction, which is virtually always present in patients for whom these new therapies are destined, are concomitantly addressed.

\section{Limitations}

Serum L-arginine levels were not measured in this trial. These may be quantified by high-performance liquid chromatography, which is cumbersome and not routinely performed clinically. Full compliance was reported by all patients, which may have resulted from the severe nature of the patients' disease, the trial's close follow-up, and the procedural involvement associated with the study. It is possible, however, that some patients may not have taken their L-arginine every day despite having reported to have done so.

Coronary endothelial function was not measured in this trial; however, L-arginine has been shown to positively affect coronary endothelial function and myocardial NO availability in large animals ${ }^{12,13}$ and humans. ${ }^{1,14}$ Because increased myocardial rather than peripheral $\mathrm{NO}$ availability was the target substrate modification in this trial, we did not perform surrogate studies of flow-mediated dilation of the brachial artery, which in addition are fraught with standardization issues. ${ }^{25}$ Other limitations of the trial include early termination and resultant randomization imbalances from doublestratification, and the lack of long-term follow-up, which will be conducted annually for the next 15 years. It is also important to note that this phase I trial was too small to fully assess the safety of L-arginine, VEGF, or their interaction, and that the observed absence of short- and mid-term complications can be interpreted as being only suggestive of safety.

\section{Conclusions}

Notwithstanding the above limitations, the phase I EMAT trial 1) brings additional insight into the mechanisms of action of new biosurgical therapies in humans by using VEGF-based angiogenesis; 2) suggests the efficacy and potential safety of L-arginine as 1 adjunct to make such therapies more effective; and 3) provides preliminary data for the design of larger clinical trials in angiogenesis and cell therapy wherein patients could potentially benefit from concomitant modification of their myocardial substrate by using L-arginine or other biologically supported and clinically safe approaches.

We acknowledge the assistance of Donna Baird, RN, Pierre Bedard, MD, Ian Burwash, MD, Greg Engel, Michael Kutryk, MD, Donna Maziak, MD, Marlie Poirier, RN, Joan Taichman, Michelle Turek, MD, Olga Walter, RN, and Kathryn Williams, MSc.

\section{References}

1. Quyyumi AA, Dakak N, Mulcahy D, Andrews NP, Husain S, Panza JA, et al. Nitric oxide activity in the atherosclerotic human coronary circulation. J Am Coll Cardiol. 1997;29:308-17.

2. Zeiher AM, Schachlinger V, Hohnloser SH, Saurbier B, Just H. Coronary atherosclerotic wall thickening and vascular reactivity in humans. Elevated high-density lipoprotein levels ameliorate abnormal vasoconstriction in early atherosclerosis. Circulation. 1994;89: 2525-32.

3. Berkenboom G, Crasset V, Giot C, Unger P, Vachiery JL, LeClerc JL. Endothelial function of internal mammary artery in patients with coronary artery disease and in cardiac transplant recipients. Am Heart J. 1998;135:488-94.

4. Ruef J, Marz W, Winkelmann BR. Markers for endothelial dysfunction, but not markers for oxidative stress correlate with classical risk factors and the severity of coronary artery disease. (A subgroup analysis from the Ludwigshafen Risk and Cardiovascular Health Study). Scand Cardiovasc J. 2006;40:274-9.

5. Ruel M, Wu GF, Khan TA, Voisine P, Bianchi C, Li J, et al. Inhibition of the cardiac angiogenic response to surgical FGF-2 therapy in a Swine endothelial dysfunction model. Circulation. 2003;108(Suppl 1):II335-40.

6. Babaei S, Teichert-Kuliszewska K, Zhang Q, Jones N, Dumont DJ, Stewart DJ. Angiogenic actions of angiopoietin-1 require endothelium-derived nitric oxide. Am J Pathol. 2003;162:1927-36.

7. Duan J, Murohara T, Ikeda H, Katoh A, Shintani S, Sasaki K, et al. Hypercholesterolemia inhibits angiogenesis in response to hindlimb ischemia: nitric oxide-dependent mechanism. Circulation. 2000;102: III370-6.

8. Kapila V, Sellke FW, Suuronen EJ, Mesana TG, Ruel M. Nitric oxide and the angiogenic response: can we improve the results of therapeutic angiogenesis? Expert Opin Investig Drugs. 2005;14:37-44.

9. O'Neill TJ 4th, Wamhoff BR, Owens GK, Skalak TC. Mobilization of bone marrow-derived cells enhances the angiogenic response to hypoxia without transdifferentiation into endothelial cells. Circ Res. 2005;97: 1027-35.

10. Fazel S, Cimini M, Chen L, Li S, Angoulvant D, Fedak P, et al. Cardioprotective c-kit + cells are from the bone marrow and regulate the myocardial balance of angiogenic cytokines. J Clin Invest. 2006; 116:1865-77.

11. Jang JJ, Ho HK, Kwan HH, Fajardo LF, Cooke JP. Angiogenesis is impaired by hypercholesterolemia: role of asymmetric dimethylarginine. Circulation. 2000;102:1414-9.

12. Voisine P, Li J, Bianchi C, Khan TA, Ruel M, Xu SH, et al. Effects of $\mathrm{L}$-arginine on fibroblast growth factor 2-induced angiogenesis in a model of endothelial dysfunction. Circulation. 2005;112:I202-7.

13. Voisine P, Bianchi C, Khan TA, Ruel M, Xu SH, Feng J, et al. Normalization of coronary microvascular reactivity and improvement in myocardial perfusion by surgical vascular endothelial growth factor therapy combined with oral supplementation of 1-arginine in a porcine model of endothelial dysfunction. J Thorac Cardiovasc Surg. 2005; 129:1414-20.

14. Lerman A, Burnett JC Jr, Higano ST, McKinley LJ, Holmes DR Jr. Long-term L-arginine supplementation improves small-vessel coronary endothelial function in humans. Circulation. 1998;97:2123-8.

15. Tousoulis D, Davies GJ, Tentolouris C, Crake T, Katsimaglis G, Stefanadis C, et al. Effects of changing the availability of the substrate for nitric oxide synthase by L-arginine administration on coronary vasomotor tone in angina patients with angiographically narrowed and in patients with normal coronary arteries. Am J Cardiol. 1998;82:1110-3, A6.

16. Lekakis JP, Papathanassiou S, Papaioannou TG, Papamichael CM, Zakopoulos N, Kotsis V, et al. Oral L-arginine improves endothelial dysfunction in patients with essential hypertension. Int $J$ Cardiol. 2002; $86: 317-23$.

17. Koifman B, Topilski I, Megidish R, Zelmanovich L, Chernihovsky T, Bykhovsy E, et al. Effects of losartan + L-arginine on nitric oxide production, endothelial cell function, and hemodynamic variables in patients with heart failure secondary to coronary heart disease. Am J Cardiol. 2006;98:172-7.

18. Ruel M, Laham RJ, Parker JA, Post MJ, Ware JA, Simons M, et al. Long-term effects of surgical angiogenic therapy with fibroblast growth factor 2 protein. J Thorac Cardiovasc Surg. 2002;124:28-34. 
19. deKemp RA, Nahmias C. Automated determination of the left ventricular long axis in cardiac positron tomography. Physiol Meas. 1996;17: 95-108.

20. Beanlands RS, Muzik O, Melon P, Sutor R, Sawada S, Muller D, et al. Noninvasive quantification of regional myocardial flow reserve in patients with coronary atherosclerosis using nitrogen- 13 ammonia positron emission tomography. Determination of extent of altered vascular reactivity. J Am Coll Cardiol. 1995;26:1465-75.

21. Schiller NB, Shah PM, Crawford M, DeMaria A, Devereux R, Feigenbaum $\mathrm{H}$, et al. Recommendations for quantitation of the left ventricle by two-dimensional echocardiography. American Society of Echocardiography Committee on Standards, Subcommittee on Quantitation of Two-Dimensional Echocardiograms. $J$ Am Soc Echocardiogr. 1989;2:358-67.

22. Yoshinaga K, Chow BJ, dekemp RA, Thorn S, Ruddy TD, Davies RA, et al. Application of cardiac molecular imaging using positron emission tomography in evaluation of drug and therapeutics for cardiovascular disorders. Curr Pharm Des. 2005;11:903-32.

23. Schulman SP, Becker LC, Kass DA, Champion HC, Terrin ML, Forman S, et al. L-arginine therapy in acute myocardial infarction: the Vascular Interaction With Age in Myocardial Infarction (VINTAGE MI) randomized clinical trial. JAMA. 2006;295:58-64.

24. Ziche M, Morbidelli L, Choudhuri R, Zhang HT, Donnini S, Granger HJ, et al. Nitric oxide synthase lies downstream from vascular endothelial growth factor-induced but not basic fibroblast growth factor-induced angiogenesis. J Clin Invest. 1997;99:2625-34.

25. Peretz A, Leotta DF, Sullivan JH, Trenga CA, Sands FN, Aulet MR, et al. Flow mediated dilation of the brachial artery: an investigation of methods requiring further standardization. BMC Cardiovasc Disord. 2007;7:11.

\section{Discussion}

Dr Todd K. Rosengart (Stony Brook, NY). The authors are to be congratulated for translating solid experimental laboratory data into a logical extension in clinical Unfortunately, as has been the case on a number of occasions, the human condition has confounded the efforts to reasonably and logically test some of your experimental hypotheses. I have a number of questions; but I'll ask you 4, which I'll ask you one at a time for simplicity.

First, why did you not administer VEGF into an unrevascularized territory so you could take advantage of a low baseline perfusion level as opposed to administering VEGF into the LAD territory? Obviously, this creates potential problems of artifact because of your bypass grafts.

Dr Ruel. This is an excellent question and a point that we had to resolve at the design level of the study. VEGF is a relatively poor angiogenic factor, but it is intimately NO dependent, perhaps more than fibroblast growth factor-2 and hepatocyte growth factor, which are other cytokines that are potentially more robust. We also chose VEGF first because it has been widely studied in humans, and part of this comes from your work as well. We also chose VEGF because it was available from our collaborators in addition to being NO dependent.

On the other hand, we did not want to expose patients to the possibility of ineffective angiogenesis, which could have happened in the VEGF and placebo groups. We thus protected our patients by bypassing all their territories as well as we could, considering that those patients all had diffuse LAD disease.

Furthermore, we had a precise measure of myocardial blood flow. By performing the anterior myocardial bypass at the same target site in every patient, which was the distal LAD, we could focus on the basal, mid, and early distal portions of the anterior wall to isolate the effect of VEGF.
In addition, the performance of baseline PET scanning, which would be repeated at 3 months, early after the operation instead of before the operation, accounted as best as possible for the confounding effects of CABG.

Dr Rosengart. You mentioned in your article and presentation that you used intraoperative validation or verification that the LAD was suitable for your experimental protocol. Can you go into a little bit more detail on that? Or conversely, did you attempt to use any kind of strict angiographic guidelines to determine whether you'd have a consistent cohort to study?

Dr Ruel. Those patients were screened on the basis of their potential ungraftability. Several of them came from other centers and were referred to us as ungraftable or likely ungraftable.

The intraoperative validation was informal. Initially, the patients were screened and found to have a diffusely diseased LAD; in many cases we thought that a LAD graft would perhaps not be constructible. As many of us know, if you really put your mind to it, you can basically graft anything with a lumen in it. So this is what we did, and we bypassed the initial healthy portion of the LAD, which in all patients corresponded to its most distal portion.

The intraoperative validation was done by a non-investigator cardiac surgeon who confirmed that each patient had diffuse distal disease, but there were no specific radiologic measures to determine eligibility for the trial. As you know, measures of coronary disease diffuseness are controversial, and despite attempts in the literature to develop those, they remain neither well accepted nor used.

Dr Rosengart. Again, in the same regard, you used baseline perfusion assay at days 3 to 5, or even in one case, I guess, day 7 postoperatively. I'd be concerned that this introduced some variability. Although you clearly show a difference between the experimental and control groups, how do you know that the change in perfusion is completely a VEGF effect as opposed to some collateralization occurring because of the bypass?

Dr Ruel. You're absolutely right. Again, your point deals with the issues of doing this study in humans. We had few alternatives. We thought that doing the baseline PET scanning preoperatively would be less precise than doing what we did. However, there are patients for whom undergoing a full PET scan 3 days after a bypass operation is not ideal, especially those, as in this population, who have diffuse coronary disease and may consequently have had a slightly longer operation.

You're absolutely right that VEGF has several effects. Angiogenic cytokines are not only angiogenic but also have acute vascular and other effects. For instance, VEGF results in local vasodilatation and increased permeability. We did see some of these effects on baseline PET scanning and tried to objectively and anatomically account for them as best as possible.

As you know, the onset of action of VEGF and other angiogenic cytokines is usually approximately 2 weeks, so we considered that the first 5 days were relatively protected from most of the angiogenic effects of the protein. It is possible that some patients may have been better responders and their effects started before.

Dr Rosengart. Can you tell us a little bit more about the toxicity, or the purported toxicity, of L-arginine? Is this a real phenomenon or how do we deal with that? If it is real, are there alternatives (which obviously is NO or nitroglycerin) that would be suitable in a clinical arena?

Dr Ruel. I think a lot of researchers are disappointed by the bad press recently attributed to L-arginine because of a single relatively 
limited scope trial published about a year ago. The trial dealt with patients who had an acute ST elevation, had no revascularization, and were randomized to receive L-arginine or not.

There may be other ways to modify endothelial function and NO bioavailability. One way, for instance, may be to up-regulate argininosuccinate synthase, which is a specific enzyme that derives endothelial NO synthase from citrulline. So there are ways other than L-arginine to make the myocardial substrate more amenable to the new therapies that we want to see become effective one day.

\section{Notice of Correction}

Kondruweit M, Weyand M, Mahmoud FO, Geißdörfer W, Schoerner C, Ropers D, Achenbach S, Strecker T. Fulminant endocarditis caused by Streptobacillus moniliformis in a young man. J Thorac Cardiovasc Surg. 2007;134:1579-80.

The spelling of the surname of author Walter Geißdörfer was incorrect. The correct spelling is shown in the author line, above. 
TABLE E1. Endothelial Modulation in Angiogenic Therapy Trial Randomization Schedule

\begin{tabular}{|c|c|c|c|c|}
\hline Patient No. (chronologic) & $\begin{array}{l}\text { Placebo/placebo } \\
\qquad(\mathbf{N}=1)\end{array}$ & $\begin{array}{c}\text { VEGF/placebo } \\
(N=7)\end{array}$ & $\begin{array}{l}\text { Placebo/L-arginine } \\
\qquad(N=6)\end{array}$ & $\begin{array}{c}\text { VEGF/L-arginine } \\
(\mathrm{N}=5)\end{array}$ \\
\hline 1 & & & $X$ & \\
\hline 2 & & & & $X$ \\
\hline 3 & & $X$ & & \\
\hline 4 & & & $X$ & \\
\hline 5 & & $X$ & & \\
\hline 6 & & & & $X$ \\
\hline 7 & & & & $X$ \\
\hline 8 & & & $X$ & \\
\hline 9 & & $X$ & & \\
\hline 10 & & & & $X$ \\
\hline 11 & & & $X$ & \\
\hline 12 & & $X$ & & \\
\hline 13 & & & & $X$ \\
\hline 14 & & $X$ & & \\
\hline 15 & & & $X$ & \\
\hline 16 & & $X$ & & \\
\hline 17 & $X$ & & & \\
\hline 18 & & $X$ & & \\
\hline 19 & & & $X$ & \\
\hline
\end{tabular}

VEGF, Vascular endothelial growth factor. 